\title{
Prediction of key gene function in spinal muscular atrophy using guilt by association method based on network and gene ontology
}

\author{
WENJIU YANG ${ }^{1}$, JING HAN $^{2}$, JINFENG MA ${ }^{1}$, YUJIE FENG ${ }^{3}$, \\ QINGXIAN HOU ${ }^{1}$, ZHIJIE WANG ${ }^{1}$ and TENGBO YU ${ }^{4}$ \\ Departments of ${ }^{1}$ Spine Surgery, ${ }^{2}$ Ophthalmology, ${ }^{3}$ Hepatobiliary Surgery and ${ }^{4}$ Sports Medicine, \\ The Affiliated Hospital of Qingdao University, Qingdao, Shandong 266071, P.R. China
}

Received June 4, 2018; Accepted January 23, 2019

DOI: 10.3892/etm.2019.7216

\begin{abstract}
Guilt by association (GBA) algorithm has been widely used to predict gene functions statistically, and a network-based approach may increase the confidence and veracity of identifying molecular signatures for diseases. The aim of the present study was to suggest a gene ontology (GO)-based method by integrating the GBA algorithm and network, to identify key gene functions for spinal muscular atrophy (SMA). The inference of predicting key gene functions was comprised of four steps, preparing gene lists and sets; extracting differentially expressed genes (DEGs) using microarray data [linear models for microarray data (limma)] package; constructing a co-expression matrix on gene lists using the Spearman correlation coefficient method; and predicting gene functions by GBA algorithm. Ultimately, key gene functions were predicted according to the area under the curve (AUC) index for GO terms and the GO terms with AUC $>0.7$ were determined as the optimal gene functions for SMA. A total of 484 DEGs and 466 background GO terms were regarded as gene lists and sets for the subsequent analyses, respectively. The predicted results obtained from the network-based GBA approach showed 141 gene sets had a good classified performance with AUC $>0.5$. Most significantly, 3 gene sets with AUC $>0.7$ were denoted as seed gene functions for SMA, including cell morphogenesis, which is involved in differentiation and ossification. In conclusion, we have predicted 3 key gene functions for SMA compared with control utilizing network-based GBA algorithm. The findings may provide great insights to reveal pathological and molecular mechanism underlying SMA.
\end{abstract}

Correspondence to: Dr Tengbo Yu, Department of Sports Medicine, The Affiliated Hospital of Qingdao University, 16 Jiangsu Road, Qingdao, Shandong 266071, P.R. China

E-mail: yutengbo1205@163.com

Key words: spinal muscular atrophy, gene ontology, guilt by association, co-expression, network, gene function

\section{Introduction}

As a major genetic cause of infant mortality, spinal muscular atrophy (SMA) is a destructive and inherited neurodegenerative disorder characterised by the loss of motor neurons in the anterior horn of the spinal cord, resulting in muscle wasting and weakness $(1,2)$. It is currently an incurable neuromuscular disorder. Survival motor neuron 1 (SMN1) gene encodes SMN, which is widely expressed in all the eukaryotic cells. Approximately $95 \%$ of SMA cases are related to deletions or mutations of the SMN1 (3). Gene therapy research has made significant progress over the past decade, and one of the rapidly emerging neurological fields is the delivery of genes to the central nervous system (CNS) through in vivo or in vitro techniques (4). In addition, a good understanding of pathological and molecular mechanism underlying SMA may offer great help to explore effective therapy of this complicated disease.

Particularly, the difference of gene expression levels could reflect the propensity of many diseases, and thus identifying gene functions has been an effective way to reveal the pathological mechanism of a disease at molecular level (5). Zeng et al used a novel correlation measure known as HeteSim in order to focus on candidate disease genes (6). Establishing a network-based approach to identify new genes that may be related to infertility is imperative (7). Furthermore, it has been demonstrated that gene function predictions can be performed with very high statistical confidence using variants based on guilt by association (GBA) algorithm, with the hypothesis that the association in genetic data is necessary to establishing guilt (8). Although various techniques have been proposed for the purpose of extending GBA to indirect connections, only slight effectiveness was identified (9-11). Consequently, treatments targeting only one gene are not always effective, because genes usually do not work alone, but co-operate with others.

Therefore, in the present study, a new method was proposed to predict key gene functions for progressive SMA patients, by integrating the GBA algorithm and network-based method. To achieve this aim, firstly, gene expression data and gene ontology (GO) annotations were collected from the public databases, respectively. Secondly, differentially expressed genes (DEGs) were identified as gene lists and background GO terms were extracted as gene sets. Thirdly, the co-expression matrix 
(CEM) was constructed on gene lists by Spearman correlation coefficient (SCC) method. Ultimately, gene functions were predicted by integrating the CEM and GBA algorithm, of which the area under the receiver operating characteristics curve (AUC) was applied to select the key gene functions in SMA patients.

\section{Materials and methods}

Preparing gene expression data. In this study, gene expression data (GSE38417) for human SMA, deposited on Affymetrix Gene Chip Human Genome HGU133 Plus 2 Array [HGU133_Plus_2], were obtained from the public-free Gene Expression Omnibus (GEO) database (https://www. ncbi.nlm.nih.gov/geo/). First, we combined multiple probes that corresponded to the same gene, and selected the average value of the plurality of probes as the expression value of the gene. Second, the annotation information was modified, the column name corresponding to the line, renamed 'groups', including control (6 samples) and SMA (16 samples). In order to control the quality of the data, standard pretreatments were performed $(12,13)$.

Identifying DEGs. During this step, DEGs between control and SMA were detected utilizing the linear models for microarray data (limma) package. In detail, the $1 \mathrm{mFit}$ function implemented in limma was utilized to perform linear fitting, empirical Bayes statistics and false discovery rate (FDR) calibration of the P-values on the data. The thresholds for DEGs were set as $\mathrm{P}<0.95$ and llog fold change $(\mathrm{FC}) \mid \geq 0.5$.

Constructing CEM. To further investigate the correlations or interactions among DEGs, a CEM for them was constructed based on the SCC algorithm. To the best of our knowledge, SCC is a main measure used to determine the correlation between two variables, and its value is between -1 and +1 inclusive. If the SCC for a pair of genes was positive, it would indicate a positive linear correlation between the two genes. Similarly, a negative SCC refers to a negative relationship of the gene pair. The absolute SCC value of an interaction was denoted as its weight value. Furthermore, the higher the weight value across two genes, the stronger the interaction was, especially for 1 . Otherwise, the 0 meant that there was no interaction between two genes. As a result, a CEM was constructed according to the weight. An assortativity coefficient was calculated to assess degree assortative mixing pattern extent. Assortativity coefficient, which is obtained by using R-package igraph, is an intuitive inspection and measurement of the positive and negative correlation of node relationships in a network. In other words, according to the positive and negative values of the network node, it can be discriminated whether it is a homogeneous cluster or a heterogeneous cluster.

GO-term enrichment analysis. Human GO annotations were prepared from the Gene Ontology Consortium (http://geneontology.org/), which is a community-based bioinformatics resource that supplies information on gene product function applying ontologies to represent biological knowledge (14). Subsequently, we propagated over the GO structure and
Table I. The top 10 DEGs.

\begin{tabular}{lcccc}
\hline Genes & $|\log (\mathrm{FC})|$ & $\mathrm{t}$ value & P-value & $\begin{array}{c}\text { Adjusted } \\
\text { P-value }\end{array}$ \\
\hline TYRP1 & -1.80647 & -51.642 & $2.65 \times 10^{-23}$ & $5.43 \times 10^{-19}$ \\
ETNPPL & -1.5344 & -28.2636 & $5.60 \times 10^{-18}$ & $5.46 \times 10^{-14}$ \\
LGI1 & -1.53461 & -27.5554 & $9.33 \times 10^{-18}$ & $5.46 \times 10^{-14}$ \\
CFAP46 & -0.6102 & -27.3737 & $1.07 \times 10^{-17}$ & $5.46 \times 10^{-14}$ \\
FZD10 & 1.417835 & 26.67597 & $1.79 \times 10^{-17}$ & $7.34 \times 10^{-14}$ \\
CRISPLD1 & 1.174577 & 26.21701 & $2.54 \times 10^{-17}$ & $7.43 \times 10^{-14}$ \\
FAM179A & -1.39983 & -25.5108 & $4.38 \times 10^{-17}$ & $1.02 \times 10^{-13}$ \\
COL3A1 & 0.503541 & 25.48533 & $4.47 \times 10^{-17}$ & $1.02 \times 10^{-13}$ \\
ERBB3 & 0.787361 & 24.45334 & $1.02 \times 10^{-16}$ & $2.10 \times 10^{-13}$ \\
SCGB1D2 & -1.48211 & -23.5802 & $2.11 \times 10^{-16}$ & $3.94 \times 10^{-13}$ \\
\hline
\end{tabular}

Based on 20,514 genes in GSE38417, we identified 484 DEGs between control and DMN by limma package when setting the thresholds as $\mathrm{P}<0.05$ and $\log \mathrm{FCl} \geq 0.5$. DEGs, differentially expressed genes; FC, fold change; limma, linear models for microarray data.

filtered for GO terms on size so that each remaining term had between 20 and 1,000 related genes, while excluding those inferred from electronic annotation $(15,16)$. For purpose of making these retained GO terms more correlated to SMA, we took the intersections between DEGs and GO terms. If the number of DEGs for a GO term was $<20$, it would be removed. In other words, only GO terms including $\geq 20$ DEGs were reserved.

Network-based GBA algorithm. As mentioned above, we combined the GBA algorithm with network to predict significant gene functions for progressors. The principle of GBA is to use relational information (e.g., interactions) to predict new gene members in the functional call of genes. Specifically, for a DEG in the CEM, we chose its adjacent genes to enrich to a GO term. Based on these $\mathrm{GO}$ functional annotations, a multi-functionality $(M F)$ score was assigned to each gene $i$ in the CEM (4):

$$
M F(i)=\sum_{k \mid i \in G o_{k}} \frac{1}{N u m_{\text {in }_{k}} * N u m_{\text {out }_{k}}}
$$

Of which $\mathrm{Num}_{i n_{k}}$ represented the number of genes within GO group $k$, whose weighing had the impact of contributing to a GO group; and Num $_{\text {out }_{\text {k }}}$ was the number of genes outside the GO group $k$ in the CEM, whose weighing provided a corresponding weight to genes not within the GO group. It should be noted that weighing referred to the effect of calculating membership of a group based on the degree of gene contribution to that GO group. Thus, 3-fold cross-validation was applied to evaluate the scoring genes ranked in the MF score to determine how well they belonged to the known gene set, and computed the AUC for evaluating classification performances between progressors and non-progressors. In the present study, to assess the predictive power of machine learners in support vector machines (SVM) model, AUC was considered a better measure than the accuracy of assessing clinical classification performance. Most importantly, an AUC of 0.5 represents 
A

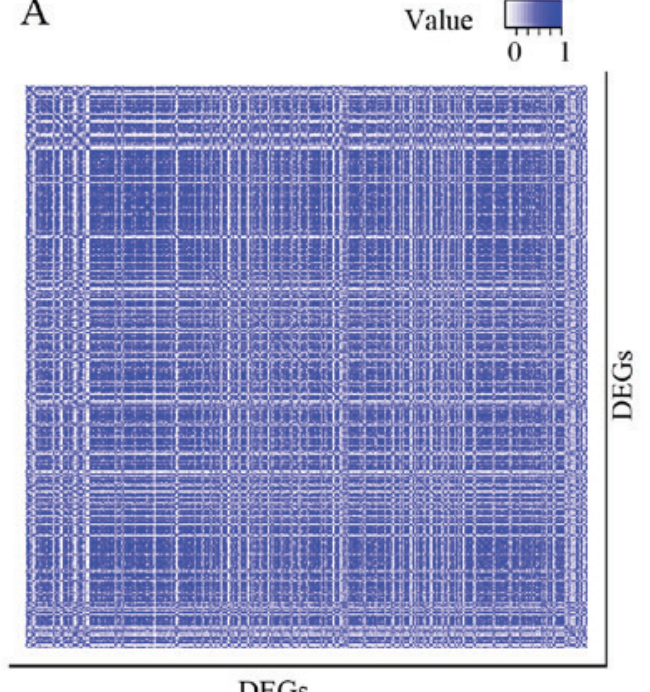

B

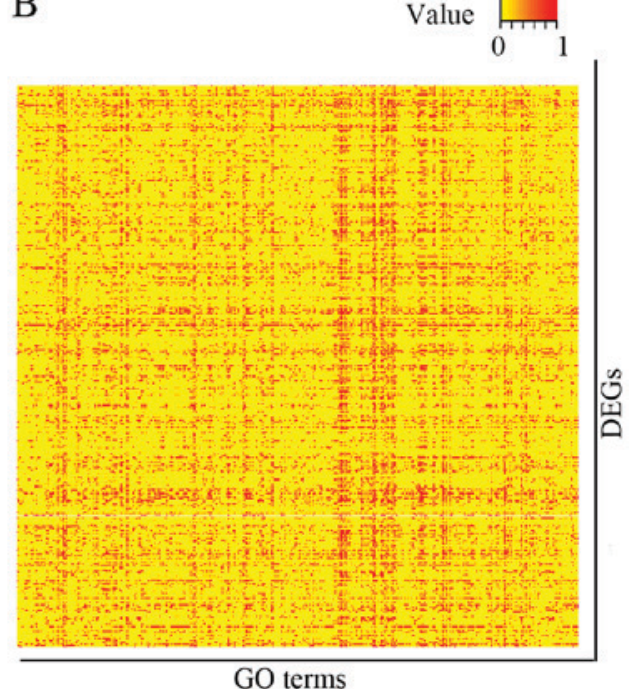

Figure 1. Two necessary objects for the network-based function inference by extending the 'guilt by association' method. (A) Differential co-expression network matrix. Heatmap clarified clustering of microarray data for 484 DEGs. The darker the blue, the stronger the relationship between the genes. The weaker the blue or the white, the less the relationship between the genes or even no relationship between them. (B) Gene set annotation vectors. The horizontal axis shows the clustering of $466 \mathrm{GO}$ terms, and the vertical axis represents the clustering of the $484 \mathrm{DEGs}$. The deeper the red, the closer the association between the gene and the GO term in the sample channel. By contrast, yellow indicates that the correlation is small between the gene and the GO term in the reference channel. DEGs, differentially expressed genes; GO, gene ontology.

classification of the level of opportunity, while an AUC of 1.0 represents the perfect classification (17). Thus, we defined GO terms of AUC $>0.7$ as key gene functions for SMA patients in the present study.

\section{Results}

Gene lists and gene sets. In the present study, differential co-expression network and GO annotations were the two main necessary objects for network-based function inference in SMA. To achieve this, we first obtained the accessible expression data of SMA to identify the DEGs and generate the gene co-expression. Based on 20,514 genes in GSE38417, we identified 484 DEGs between control and DMN by limma package when setting the thresholds as $\mathrm{P}<0.05$ and $\mid \log \mathrm{FCl} \geq 0.5$. The top 10 DEGs are shown in Table I. Then based on these DEGs, the SCC values among gene pairs were calculated, and the SCC absolute values were defined as the weight values. We constructed the co-expression adjacency matrix of 484 DEGs (covering 116,886 interactions), where the entry indicated the connection between two genes (Fig. 1A). Only gene sets containing intersected DEGs of $>20$ were left in the subsequent analysis. Thereafter, we defined the amount of intersected DEGs as the count value of this term. As a result, a total of $466 \mathrm{GO}$ terms were determined. Subsequently, these GO terms were represented as a binary vector, where each entry corresponded to a differentially expressed gene, with a 1 indicating that the differentially expressed gene was a member of this GO term, and 0 if it was not (Fig. 1B).

CEM. To investigate biological correlations among DEGs, a CEM with 484 nodes and 116,886 interactions was constructed based on the SCC, of which each interaction possessed a weight value to reveal the interacted strength between two genes. The weight distribution for interactions in this CEM

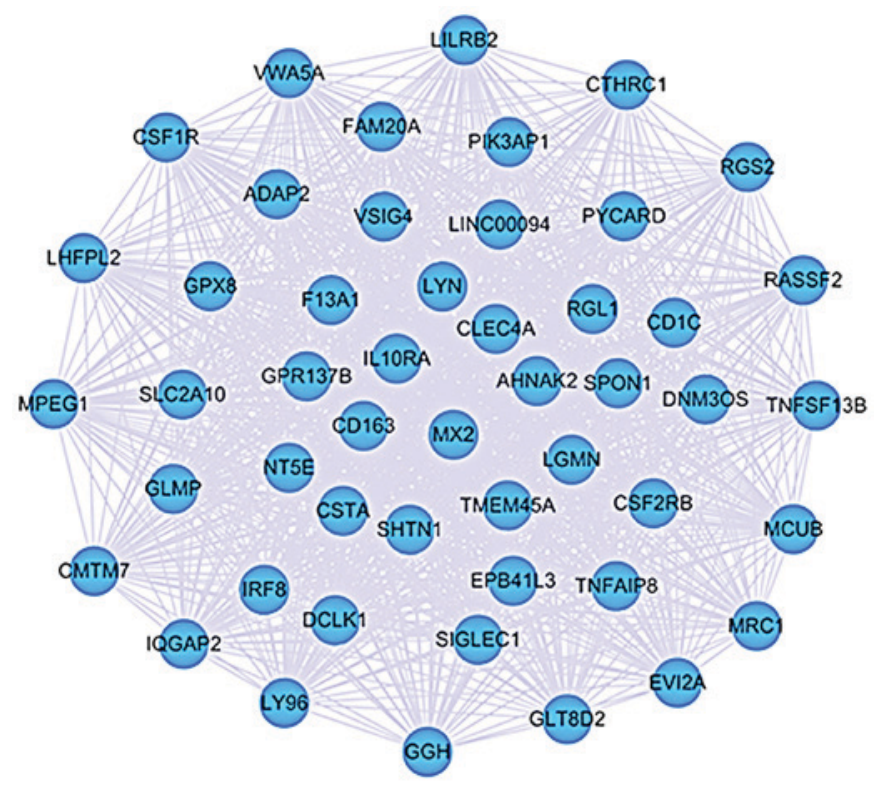

Figure 2. Sub-network of CEM. There were 485 nodes and 1,146 edges, of which nodes were on behalf of DEGs and edges represented interactions between two DEGs with weight $>0.8$. CEM, co-expression matrices; DEGs, differentially expressed genes.

showed the characteristic of good adjacent matrix that weighs on its diagonal was nearly equal to 1 , which suggested that the CEM had a good network scale property. The greater the weight value, the more likely the interaction was related to the occurrence of disease. In particular, an edge between RASSF 2 and LHFPL2 had the highest weight of 0.998, which indicated that the edge or the two genes may be associated with SMA. In addition, to further evaluate the activities of genes in interactions of high weights, topological degree centrality analysis was conducted on all the nodes in the CEM. The assortativity 

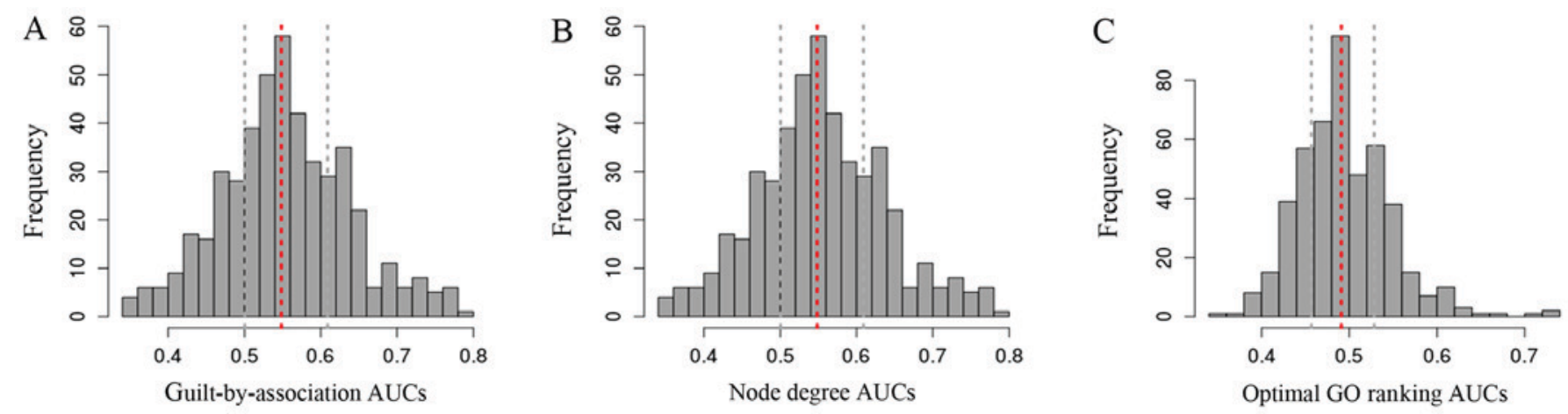

Figure 3. Distributions of the area under the receiver operating characteristics curve (AUC) scores. (A) Distribution of AUC scores from the neighbor voting algorithm. (B) Distribution of AUC scores for node degree ranking. (C) Distribution of AUC scores from multifunctionality assessment. Red, median; grey, inter-quartile ranges. AUC, area under the curve.

Table II. Optimal gene functions for SMA.

\begin{tabular}{lllc}
\hline ID & \multicolumn{1}{c}{ Term } & Domain & AUCs \\
\hline GO:0000902 & Cell morphogenesis & Biological_process & 0.724619 \\
GO:0000904 & $\begin{array}{l}\text { Cell morphogenesis } \\
\text { involved in } \\
\text { differentiation }\end{array}$ & Biological_process & 0.724619 \\
GO:0001503 & Ossification & Biological_process & 0.703075 \\
\hline
\end{tabular}

Among 466 GO terms, a total of 141 terms were identified under AUC $>0.5$. Based on the optimal ranking gene list, GO terms with AUC $>0.7$ were defined as the optimal gene functions for SMA. AUC, area under the curve; GO, gene ontology; SMA, spinal muscular atrophy.

coefficient for the CEM was 0.393 , indicating that the network had perfect assortative mixing patterns.

Generally speaking, a network with too many interactions might be too generic and interactions with low weight are not as significant as high ones. Thus, we extracted a sub-network from the CEM by selecting those interactions with weight $>0.8$ and visualized it by Cytoscape software (Fig. 2). There were 485 nodes and 1,146 edges, of which nodes were on the behalf of DEGs and edges represented interactions between two DEGs with weight $>0.8$.

Gene function inference. Generally, genes with similar neighbors may share common properties. Thus we performed the gene function inference for SMA based on the differential co-expression network. Firstly, neighbor voting algorithm was employed to perform the gene function prediction by integrating differential co-expression network and GO annotations. The AUC value was implemented to determine the performance metric for each GO annotation, and GO terms with AUC $>0.7$ was regarded as candidate gene functions. The distribution of AUC scores from the neighbor voting algorithm is shown in Fig. 3. Among 466 GO terms, 141 terms were identified under AUC $>0.5$. By calculating the multifunctionality score for each DEG, we generated a list of genes ranked by multifunctionality score, i.e., the optimal ranking gene list. Based on the optimal ranking gene list, GO terms with AUC $>0.7$ were defined as the optimal gene functions for SMA, including cell morphogenesis $(\mathrm{AUC}=0.724)$, cell morphogenesis involved in differentiation $(\mathrm{AUC}=0.724)$, ossification (AUC $=0.703$ ). The details are shown in Table II.

\section{Discussion}

In the present study, we aimed to predict key gene functions in SMA patients by using a network-based GBA method, since the network-based approach could systematically investigate the molecular complexity of a particular disease and identify potential signatures through bio-molecular networks rather than individual genes (18). Simultaneously, network co-expressed analysis enhances the statistical confidence of individual connections, increases overlap with protein interaction, and take advantage of mathematical convenience. Moreover, previous studies have shown that GBA variants can ingrease statistical confidence in predicting gene function, assuming that the associations in the data of a gene are necessary in establishing guilt (19). Notably, an integration of co-expression network and the GBA algorithm would provide a new manner to predict significant gene functions and reveal molecular mechanism underlying SMA.

On the basis of SCC method, a CEM for progressors was constructed on DEGs, and further a sub-network of weight $>0.8$ was extracted from the CEM. Of note, we found that TYRPI, ETNPPL and another top 10 genes had high degree both in CEM and its sub-network, which indicated their importance as progressors. The groups of Becker et al and Rousseau et al thought that the dys-regulation of TYRPI may disturb the normal development of cells and tissues, resulting in brain damage and even nervous system injury $(20,21)$. Veiga-daCunha et al suggest that the ETNPPL-mediated degradation of ethanolaminephosphate could balance the concentration of that metabolite, which might contribute to mental disorders such as schizophrenia was in the CNS (22). LGII is mainly used for cognitive dysfunction and seizures, and is mostly expressed in neurons and serum LGII autoantibodies in patients with limbic encephalitis (LE) $(23,24)$. In line with these findings, the expression of three genes was associated with nervous system injury, which play an important role in SMA.

The rest of the genes do not appear to be related to SMA or nerves, for instance, FZD10 is the receptor for Wnt molecules and is extensively involved in various cell processes $(25,26)$; Crispldl acts in tissue culture models of osteoarthritis (27); 
FAM179A plays a role in detecting ALK fusion of patients with non-small cell lung cancer (28); COL3A1 encodes the pro- $\alpha 1$ chains of type III collagen, which is found in extensible connective tissues such as uterus, skin, lung, intestine and the vascular system (29); ERBB3 has a neuregulin binding domain (30). To the best of our knowledge, this is the first study to reveal the key role of these genes in human SMA patients, which could be useful in future research in treatment and prediction of SMA.

Particularly, 466 background GO terms were identified as gene sets for the present study. Most significantly, 3 gene sets with AUC >0.7 were denoted as key gene functions for SMA, including cell morphogenesis, cell morphogenesis involved in differentiation and ossification. In detail, as the development of shape or morphologies in cells or organisms, morphogenesis is a fundamental but poorly understood process throughout biology $(31,32)$. SMA is a motor neuron disease that degenerates the spinal cord and muscle. Studis confirmed that there are some in vivo benefits of intrathecal injection of neural stemcell in severe SMA mice following differentiation of neural stem cells to alter cell morphogenesis from mouse spinal cord neurospheres $(33,34)$. Therefore, our finding has important implications for the molecular mechanisms of SMA.

Besides, molecular function represents elemental activities, such as differentiation and ossification (35), describing the actions of a gene product at the molecular level. It is a common phenomenon that a given gene presents in one or more molecular functions (such as TYRP1 described above), and two or more genes exhibit the same one function $(36,37)$. Genes with similar functions observe similar annotation patterns in their neighborhood, regardless of the distance between them in the interaction network. Using single-stranded DNA oligonucleotides into the cells, Anderton and Mastaglia (34) induced a genome editing of SMN2 at the molecular level, thus modifying the SMA-iPSC-derived motor neurons. Upon direct transplantation into a severe SMA mouse model, muscle connections and ossification were all ameliorated $(34,38)$.

In conclusion, we have predicted 3 seed gene functions for SMA compared with control utilizing network-based GBA algorithm. The findings might give great insights to reveal pathological and molecular mechanism underlying SMA. However, the expression data used in this work was recruited from the open access database, and the 3 seed gene functions are not validated. In future research, these validations should be performed. Of note, we are preparing the microarray data ourselves at present and further study should be conducted on the role these validations are to play.

\section{Acknowledgements}

Not applicable.

\section{Funding}

No funding was received.

\section{Availability of data and materials}

The datasets used and/or analyzed during the present study are available from the corresponding author on reasonable request.

\section{Authors' contributions}

WY and JH conceived the study and drafted the manuscript. JM and YF acquired the data; QH, ZW and TY analyzed the data and revised the manuscript. All authors read and approved the final study.

\section{Ethics approval and consent to participate}

Not applicable.

\section{Patient consent for publication}

Not applicable.

\section{Competing interests}

The authors declare that they have no competing interests.

\section{References}

1. Nurputra DK, Lai PS, Harahap NI, Morikawa S, Yamamoto T, Nishimura N, Kubo Y, Takeuchi A, Saito T, Takeshima Y, et al: Spinal muscular atrophy: From gene discovery to clinical trials. Ann Hum Genet 77: 435-463, 2013.

2. Grunseich C, Zukosky K, Kats IR, Ghosh L, Harmison GG, Bott LC, Rinaldi C, Chen KL, Chen G, Boehm M, et al: Stem cell-derived motor neurons from spinal and bulbar muscular atrophy patients. Neurobiol Dis 70: 12-20, 2014.

3. Kirschner J, Schorling D, Hauschke D, Rensing-Zimmermann C, Wein U, Grieben U, Schottmann G, Schara U, Konrad K, Müller-Felber W, et al: Somatropin treatment of spinal muscular atrophy: A placebo-controlled, double-blind crossover pilot study. Neuromuscul Disord 24: 134-142, 2014.

4. Gillis $J$ and Pavlidis P: The impact of multifunctional genes on 'guilt by association' analysis. PLoS One 6: e17258, 2011.

5. Gillis J and Pavlidis P: 'Guilt by Association' is the exception rather than the rule in gene networks. PLoS Comput Biol 8: e1002444, 2012.

6. Zeng X, Liao Y, Liu Y and Zou Q: Prediction and validation of disease genes using HeteSim Scores. IEEE/ACM Trans Comput Biol Bioinformatics 14: 687-695, 2017.

7. Wang S, Huang G, Hu Q and Zou Q: A network-based method for the identification of putative genes related to infertility. Biochim Biophys Acta 1860 (11 Pt B): 2716-2724, 2016.

8. Molet M, Stagner JP, Miller HC, Kosinski T and Zentall TR: Guilt by association and honor by association: The role of acquired equivalence. Psychon Bull Rev 20: 385-390, 2013.

9. Vey G: Metagenomic guilt by association: An operonic perspective. PLoS One 8: e71484, 2013.

10. Mulcahy PJ, Iremonger K, Karyka E, Herranz-Martín S, Shum KT, Tam JK and Azzouz M: Gene therapy: A promising approach to treating spinal muscular atrophy. Hum Gene Ther 25: 575-586, 2014.

11. Raleigh DP: Guilt by association: The physical chemistry and biology of protein aggregation. J Phys Chem Lett 5: 2012-2014, 2014.

12. Verleyen W, Ballouz S and Gillis J: Measuring the wisdom of the crowds in network-based gene function inference. Bioinformatics 31: 745-752, 2014.

13. Levy PD: Guilt by association - A closer look at calcium, heart failure, and mortality. J Card Fail 21: 628-629, 2015.

14. Ashburner M, Ball CA, Blake JA, Botstein D, Butler H, Cherry JM, Davis AP, Dolinski K, Dwight SS, Eppig JT, et al; The Gene Ontology Consortium: Gene ontology: Tool for the unification of biology. Nat Genet 25: 25-29, 2000.

15. Huntley RP, Harris MA, Alam-Faruque Y, Blake JA, Carbon S, Dietze H, Dimmer EC, Foulger RE, Hill DP, Khodiyar VK, et al: A method for increasing expressivity of Gene Ontology annotations using a compositional approach. BMC Bioinformatics 15: 155,2014

16. Gene Ontology Consortium: Gene Ontology Consortium: Going forward. Nucleic Acids Res 43 (D1): D1049-D1056, 2015. 
17. Zou M, Liu Z, Zhang XS and Wang Y: NCC-AUC: An AUC optimization method to identify multibiomarker panel for cancer prognosis from genomic and clinical data. Bioinformatics 15 : 3330-3338, 2015.

18. Gillis J and Pavlidis P: The role of indirect connections in gene networks in predicting function. Bioinformatics 27: 1860-1866, 2011.

19. Willson DF: Outcomes and risk factors in pediatric ventilator-associated pneumonia: Guilt by association. Pediatr Crit Care Med 16: 299-301, 2015.

20. Becker D, Otto M, Ammann P, Keller I, Drögemüller C and Leeb T: The brown coat colour of Coppernecked goats is associated with a non-synonymous variant at the TYRP1 locus on chromosome 8. Anim Genet 46: 50-54, 2015.

21. Rousseau B, Larrieu-Lahargue F, Javerzat S, Guilhem-Ducléon F Beermann F and Bikfalvi A: The tyrp1-Tag/tyrp1-FGFR1-DN bigenic mouse: a model for selective inhibition of tumor development, angiogenesis, and invasion into the neural tissue by blockade of fibroblast growth factor receptor activity. Cancer Res 64: 2490-2495, 2004

22. Veiga-da-Cunha M, Hadi F, Balligand T, Stroobant V and Van Schaftingen E: Molecular identification of hydroxylysine kinase and of ammoniophospholyases acting on 5-phosphohydroxy-L-lysine and phosphoethanolamine. J Biol Chem 287: 7246-7255, 2012.

23. Vives-Rodriguez A, Sivaraju A and Louis ED: Drop attacks: A clinical manifestation of LGI1 encephalitis. Neurol Clin Pract 7 : 442-443, 2017.

24. Nikolaus M, Jackowski-Dohrmann S, Prüss H, Schuelke M and Knierim E: Morvan syndrome associated with CASPR2 and LGI1 antibodies in a child. Neurology 90: 183-185, 2018

25. Chen Y, Huang Q, Zhou H, Wang Y, Hu X and Li T: Inhibition of canonical WNT/ $\beta$-catenin signaling is involved in leflunomide (LEF)-mediated cytotoxic effects on renal carcinoma cells. Oncotarget 7: 50401-50416, 2016.

26. Chen Z, Gao Y, Yao L, Liu Y, Huang L, Yan Z, Zhao W, Zhu P and Weng H: LncFZD6 initiates Wnt/ $\beta$-catenin and liver TIC self-renewal through BRG1-mediated FZD6 transcriptional activation. Oncogene 37: 3098-3112, 2018.

27. Wan Y, Rogers MB and Szabo-Rogers HL: A six-gene expression toolbox for the glands, epithelium and chondrocytes in the mouse nasal cavity. Gene Expr Patterns 27: 46-55, 2018

28. Cui S, Zhang W, Xiong L, Pan F, Niu Y, Chu T, Wang H, Zhao Y and Jiang L: Use of capture-based next-generation sequencing to detect ALK fusion in plasma cell-free DNA of patients with non-small-cell lung cancer. Oncotarget 8: 2771-2780, 2017.
29. Shojaati G, Khandaker I, Sylakowski K, Funderburgh ML, Du Y and Funderburgh JL: Compressed collagen enhances stem cell therapy for corneal scarring. Stem Cells Transl Med 7: 487-494, 2018.

30. Drilon A, Somwar R, Mangatt BP, Edgren H, Desmeules P, Ruusulehto A, Smith RS, Delasos L, Vojnic M, Plodkowski AJ, et al: Response to ERBB3-directed targeted therapy in NRG1-rearranged cancers. Cancer Discov 8: 686-695, 2018.

31. Varner VD and Nelson CM: Cellular and physical mechanisms of branching morphogenesis. Development 141: 2750-2759, 2014.

32. Wu M and Li J: Numb family proteins: Novel players in cardiac morphogenesis and cardiac progenitor cell differentiation. Biomol Concepts 6: 137-148, 2015.

33. Kolb SJ and Kissel JT: Spinal muscular atrophy. Neurol Clin 33: 831-846, 2015.

34. Anderton RS and Mastaglia FL: Advances and challenges in developing a therapy for spinal muscular atrophy. Expert Rev Neurother 15: 895-908, 2015.

35. Takahashi K, Satoh M, Takahashi Y, Osaki T, Nasu T, Tamada M, Okabayashi H, Nakamura M and Morino Y: Dysregulation of ossification-related miRNAs in circulating osteogenic progenitor cells obtained from patients with aortic stenosis. Clin Sci (Lond) 130: 1115-1124, 2016

36. Li J, Jiang D, Zhou H, Li F, Yang J, Hong L, Fu X, Li Z, Liu Z, Li J, et al: Expression of RNA-interference/antisense transgenes by the cognate promoters of target genes is a better gene-silencing strategy to study gene functions in rice. PLoS One 6: e17444, 2011.

37. Klie S, Nikoloski Z and Selbig J: Biological cluster evaluation for gene function prediction. J Comput Biol 21: 428-445, 2014.

38. Zanetta C, Nizzardo M, Simone C, Monguzzi E, Bresolin N, Comi GP and Corti S: Molecular therapeutic strategies for spinal muscular atrophies: Current and future clinical trials. Clin Ther 36: 128-140, 2014

This work is licensed under a Creative Commons Attribution-NonCommercial-NoDerivatives 4.0 International (CC BY-NC-ND 4.0) License. 